\title{
La temporalité du projet d'aménagement à l'épreuve des dilemmes éthiques liés à la participation citoyenne
}

\author{
Manel Djemel \\ M. Sc. Architecture, M. Urbanisme et Candidate au Ph. D. en aménagement à la Faculté de \\ l'aménagement de l'Université de Montréal \\ Email : manel.djemel@umontreal.ca
}

Machelle Ye

Architecte, Urbaniste et Candidate au Ph. D. en aménagement à la Faculté de l'aménagement de l'Université de Montréal

Email : hayazouma.fati.machelle.ye@umontreal.ca

Rabah Bousbaci

Professeur agrégé à l’École de design, de la Faculté de l'aménagement à l’Université de Montréal Email : rabah.bousbaci@umontreal.ca

Sibe Web : <https://amenagement.umontreal.ca/professeurs/fiche/in/in15051/sg/Rabah\%2oBousbaci>

\section{Gonzalo Lizarralde}

Professeur à l'École d'Architecture de la Faculté de l'aménagement à l'Université de Montréal, Titulaire de la Chaire Fayolle-Magil Construction en architecture, bâtiment et durabilité. Directeur du Groupe de recherche IF (grif) et Euvre durable

Email : gonzalo.lizarralde@umontreal.ca

Site web : <https://amenagement.umontreal.ca/professeurs/fiche/in/in14894/sg/Gonzalo\%20

Lizarralde>

\section{Résumé}

Les projets d'aménagement urbain mobilisent un réseau complexe de parties prenantes (PP) qui interviennent à plusieurs échelles et niveaux décisionnels du projet. Ces PP défendent des intérêts différents et parfois divergents. Les travaux de recherche sur les acteurs de l'aménagement ont montré l'émergence de nouveaux paradigmes qui ont une influence significative sur les processus de prise de décision. Parmi ces derniers, la participation citoyenne (PC), présentée comme une modalité qui vise la « démocratie directe » et la " justice sociale », et à favoriser l'acceptabilité sociale des projets. Paradoxalement, la PC devient parfois une source de conflits et de controverses, entrainant de ce fait la complexification voire le ralentissement des processus de projet.

L'objet de cet article est de comprendre l'influence des conflits et controverses liés à la PC sur les temporalités du projet. Nous aborderons ce phénomène sous l'angle de l'éthique, entendue comme élément moteur de la justice sociale. À partir de l'analyse d'un projet de construction d'une école primaire dans un arrondissement de la ville de Montréal, l'observation du phénomène sous la lentille des théories éthiques nous permet de mettre en lumière les principales controverses découlant de la PC et leurs effets sur le temps de réalisation du projet. L'analyse montre que la démarche participative, qui était mise en place au départ dans une perspective d'inclusion sociale et d'expérimentation de nouvelles cultures de projet, a mené à un long processus de prise de décision.

\section{Mots-clés}

Projet d'aménagement urbain, Participation citoyenne (PC), Éthique, Parties prenantes (PP), temps, conception, conduite de projets. 
M. Djemel, M. Ye, R. Bousbaci, G. Lizarralde I La temporalité du projet d'aménagement I DOI: 10.12977/ocula2019-4

\begin{abstract}
The project's temporality facing the ethical dilemmas of citizen participation - Urban development projects mobilize a complex network of stakeholders (PP) that intervene at several scales and decision-making levels of the project. These PPs represent different and sometimes divergent interests. Research on development actors has shown the emergence of new paradigms that have a significant influence on decision-making processes. Among these, citizen participation (CP), presented as a modality that aims for "direct democracy" and "social justice", and to promote the social acceptability of projects. Paradoxically, CP sometimes becomes a source of conflict and controversy, leading to complexification or even slowing of project processes. The purpose of this article is to understand the influence of CP-related conflicts and controversies on project timelines. We will approach this phenomenon from the angle of ethics, understood as the driving force of social justice. Based on the analysis of a construction project for a primary school in a borough of the city of Montreal, the observation of the phenomenon under the lens of ethical theories allows us to highlight the main controversies stemming from the $\mathrm{CP}$ and their effects on the time of project completion. The analysis shows that the participatory approach, which was initially put in place from the perspective of social inclusion and experimentation of new project cultures, led to a long decision-making process.
\end{abstract}

\title{
Keywords
}

Urban Planning Project, Citizen Participation (CP), Ethics, Stakeholders (PP), Time, Design, Project Management.

\section{Sommaire / Content}

1. Introduction

2. Cadre conceptuel

3. Les temporalités du projet d'aménagement et le processus de prise de décision

4. Méthode : l'étude de cas d'une école primaire à l'île des sœurs à Montréal (Canada)

5. Résultats

6. L'influence du rôle des parties prenantes sur la temporalité du projet

7. Discussion

8. Conclusion

Bibliographie 


\section{Introduction}

Les disciplines de l'aménagement ont de tout temps aspiré à produire des espaces physiques toujours meilleurs. L'histoire de l'urbanisme et de l'architecture révèle en effet plusieurs courants de pensée tels que les courants hygiénistes de la fin du XIXe siècle en Europe ou la Cité-Jardin (cf. Howard 1965) théorisée par l'anglais Eben-Ezer Howard, les théories du zonage développées au cours de la période moderne, entre autres. Plus récemment, plusieurs paradigmes empruntés à d'autres champs disciplinaires et appliqués à l'aménagement se sont inscrits dans cette dynamique qui vise à « bien faire » la ville. Parmi ces derniers figurent, entre autres, le développement durable, l'innovation, la participation citoyenne, l'efficience, la résilience, etc. Sans se focaliser sur les éléments physiques de l'aménagement urbain, ces paradigmes interrogent aussi, et au même titre, les meilleurs processus et démarches qui conduisent à prendre des décisions relatives à ces espaces. Cependant, la littérature montre une certaine inefficacité du recours à ces paradigmes face aux nombreux défis majeurs du développement urbain dans le monde. Alors, et pour mieux comprendre l'enjeu sous-jacent de ces paradigmes, nous faisons l'hypothèse que les considérations éthiques s'imposent aussitôt qu'on vise le « bien » (cf. Aristote \& Tricot 1983). En effet, l'action de bien faire et de bien agir, ou tout simplement le « bien » est une notion subjective et peut, de ce fait, susciter de la controverse.

L'objet de cet article est d'exposer et ensuite comprendre les enjeux éthiques à l'origine du conflit suite à la participation citoyenne dans le processus du projet d'aménagement. Nous étudierons ensuite les influences que ces dernières peuvent avoir sur les temporalités du projet et les différentes formes d'apprentissages qu'elles ont suscitées. L'article présente l'analyse du processus d'un projet de construction d'une école primaire sur L'Île-des-Sœurs à Montréal (Canada). Le projet a fait l'objet de deux consultations publiques menées par l'OCPM (Office de la consultation publique à Montréal) et a suscité de nombreuses controverses, d'abord sur l'utilité de la construction d'une nouvelle école, puis sur le choix d'emplacement même de cette dernière. En effet, les parties prenantes du projet ont éprouvé d'énormes difficultés à avoir une vision commune sur ces sujets et le projet a fait l'objet de fortes tensions et négociations (cf. Djemel, El-Bache, Marin-Valencia \& Rivera 2015).

Nous présentons dans un premier temps le cadre conceptuel mobilisé pour poser le problème, en faisant un rappel des enjeux autour des notions de projet d'aménagement, de parties prenantes, de participation citoyenne et d'éthiques. Nous précisons ensuite le contexte du cas à l'étude en présentant le contexte urbain et géographique, les enjeux autour du projet de la nouvelle école et les principales parties prenantes mobilisées. La section suivante développe les principaux résultats de l'analyse (1) en faisant une lecture de la position des différentes catégories de parties prenantes à la lumière des courants de l'éthique ; (2) puis en précisant l'influence que la confrontation 
M. Djemel, M. Ye, R. Bousbaci, G. Lizarralde I La temporalité du projet d'aménagement I DOI: 10.12977/ocula2019-4

des positions a pu avoir sur la temporalité du projet. Nous concluons par les implications théoriques et pratiques que les résultats ont sur la participation citoyenne et le processus de prise de décision.

\section{Le cadre conceptuel}

\subsection{Le projet d'aménagement urbain}

Les projets d'aménagement sont des " opérations de régénération urbaine visant à requalifier des "morceaux" de ville [...] et à doter la ville d'équipements de prestige lui permettant de se positionner favorablement dans la compétition urbaine " (Pinson 2009:620 ; Viel et al. $2012: 1$ ). Ils font intervenir un nombre important d'acteurs et d'organisations dans un système social complexe que plusieurs auteurs ont qualifié de multi-organisation temporaire (MOT) (cf. Green, 1996). Ce terme renvoie à la fois au caractère pluridisciplinaire du système d'acteurs (cf. Rowlinson et McDermott 1999 ; Cherns \& Bryant 1984) et à la durée du projet, à la fin duquel les membres se séparent » (Lizzarlde \& Djemel 2010 : 79) .

Les projets d'aménagement mobilisent en particulier des parties prenantes (PP) dont les logiques et les intérêts sont différents, parfois divergents. Si en Europe (et particulièrement en France), ils sont principalement initiés par les instances publiques - notamment les collectivités locales - et gouvernementales, dans le monde anglo-saxon, dont le Canada, plusieurs d'entre eux sont à l'initiative de promoteurs privés (cf. Raynaud 2014). Nous nous intéresserons cependant, dans le cadre de cet article, aux projets portés par les instances publiques, pour lesquels nous présupposons un enjeu d'intérêt public susceptible de mobiliser les citoyens.

\subsection{Les parties prenantes}

Une partie prenante désigne un individu ou un groupe d'individus porteurs d'un intérêt dans une décision particulière selon le PmBok (2013 Le PmBok est le Project Management Body of Knowledge : le guide du Project Management Institute qui définit les champs de connaissance couvrant la gestion des projets). Il s'agit de personnes ou de toute entité susceptible d'affecter la décision, ou d'être affectées par cette dernière (cf. Hemmati 2002 ; Aapaoja 2014 ; Freeman 1984). Le terme est emprunté à l'anglais stakeholders et insiste sur le fait que le projet peut être influencé par un ensemble de personnes au-delà des acteurs directement impliqués dans la démarche.

Il existe plusieurs façons d'appréhender les parties prenantes dans un projet d'aménagement. Elles peuvent être regroupées en typologies d'acteurs, entre autres, selon leur statut juridique ou leur rôle sociopolitique dans le système de prise de décision (Gardesse 2011). Cette dernière catégorisation distingue (1) les acteurs politiques, porteurs et responsables de la décision (2) les professionnels de l'aménagement qui mettent en œuvre la décision et (3) les citoyens/habitants dont l'influence sur la décision dépend en grande partie de la place accordée par les politiques et les professionnels. Elle nous 
M. Djemel, M. Ye, R. Bousbaci, G. Lizarralde I La temporalité du projet d'aménagement I DOI: 10.12977/ocula2019-4

semble particulièrement pertinente pour analyser les enjeux de la participation citoyenne dans une démarche de projet puisqu'elle identifie les citoyens/ habitants/société civile comme une catégorie d'analyse du système d'acteurs, même si ces derniers se mobilisent spontanément pour prendre part au processus de projet, à défaut d'y être intégrés par les acteurs.

Tableau 1. Catégories de parties prenantes selon leur place dans le système décisionnel (Source : M. Yé), d'après Gardesse, (2011).

\begin{tabular}{|c|c|c|}
\hline Politiques & $\begin{array}{l}\text { Professionnels de } \\
\text { l'aménagement }\end{array}$ & $\begin{array}{l}\text { Société civile, habitants, } \\
\text { citoyens }\end{array}$ \\
\hline $\begin{array}{l}\text { Personnes « en position de décider } \\
\text { ou de représenter et d’expliquer la } \\
\text { décision ». } \\
\text { (Gardesse, 2011, 40). }\end{array}$ & $\begin{array}{l}\text { Personnes dont le rôle est de } \\
\text { "mettre en œuvre les orientations } \\
\text { définies par les politiques » } \\
\text { (Idt, 2009; Gardesse, 2011). }\end{array}$ & $\begin{array}{l}\text { Rôle dans le processus de projet } \\
\text { d'aménagement, fonction de la } \\
\text { façon dont les acteurs politiques et } \\
\text { professionnels les considèrent }\end{array}$ \\
\hline $\begin{array}{l}\text { Élus, leurs conseillers proches et } \\
\text { les membres de leurs cabinets. } \\
\text { (Gardesse, 2011, 40). }\end{array}$ & $\begin{array}{l}\text { Deux types } \\
\text { - Membres de structures privées } \\
\text { (bureaux d'études en urban- } \\
\text { isme et agences d'aménagement } \\
\text { et d'architecture) } \\
\text { - Personnel « technico-adminis- } \\
\text { tratif» de la municipalité. }\end{array}$ & $\begin{array}{l}\text { Individus, groupes, associations ou } \\
\text { organisations }\end{array}$ \\
\hline
\end{tabular}

\subsection{La participation citoyenne (PC)}

La PC désigne « toute activité par laquelle un(e) citoyen(ne) contribue à la définition et à la concrétisation de ce qui représente l'intérêt public et le bien commun dans une société » (Venne dans Côté et al. 2011). Il s'agit donc d'une démarche qui fournit aux citoyens (individuellement, ou en groupe) la possibilité de prendre part au processus de prise de décision, voire de contribuer de manière effective à cette dernière. Cependant, plusieurs travaux sur la PC ont montré que la mobilisation des citoyens n'entraine pas de facto leur réelle influence sur les décisions. Certains auteurs se sont attelés à classifier différents échelons de la participation en fonction du pouvoir effectif des citoyens dans la prise de décision (Arnstein 1969 ; OCDE 2002 ; Thibault, Lequin, Tremblay 2000 ; IAP2 2007 ; INM 2013). D’autres travaux ont analysé de manière concrète le rôle et la portée des dispositifs participatifs dans le processus décisionnel d'un projet d'aménagement urbain (Gardesse, 2011). Gardesse conclut par exemple que les dispositifs participatifs qu'elle analyse sont en réalité des " processus d'information du public et de consultation des habitants-citoyens les plus organisés » (Gardesse 2011 : 530). Ces dispositifs ne favorisent pas un véritable dialogue entre les parties prenantes en vue de l'élaboration du projet.

Si ces dernières conclusions entrent en contradiction avec le postulat d'une démarche négociée du projet d'aménagement, qui vise entre autres la " démocratie directe » et la « justice sociale ». Plusieurs situations de projet ont fourni des exemples qui laissent entrevoir des formes d'excès de la participation. En effet, la forte influence des citoyens dans la prise de décision conduit parfois au blocage voire à l'interruption de certains projets d'aménagement, comme ce fut le cas pour le projet du Cirque du Soleil, ou plus récemment (en 
M. Djemel, M. Ye, R. Bousbaci, G. Lizarralde I La temporalité du projet d'aménagement I DOI: 10.12977/ocula2019-4

2017) pour le technopôle Angus, tous les deux à Montréal. Ces situations nous conduisent notamment à réinterroger les enjeux de la participation citoyenne sous l'angle de l'éthique ; faut-il à tout prix faire participer les citoyens en courant le risque d'interrompre un projet qui, à première vue servirait l'intérêt public et le bien commun ? Quels intérêts et points de vue faut-il privilégier dans la prise de décision? Quelle est la bonne action à poser par chacun?

\subsection{L'éthique}

L'éthique est une notion qui suscite davantage d'intérêt depuis quelques années, entre autres au Québec. Elle renvoie, d'après la commission de l'éthique en science et en technologie (CEST) à des idéaux (principes et valeurs) auxquels l'on se réfère pour poser une action que l'on estime bonne. Si pour certains le terme s'apparente à la morale en faisant référence aux racines, respectivement grecque (ethos) et latine (mores) qui signifient mœurs, d'autres font une distinction nette du sens que portent ces deux termes (CEST 2017). La morale ferait référence à un « ensemble de principes et valeurs [...] auxquels il faudrait se conformer ${ }^{1}$ » tandis que l'éthique serait une « réflexion argumentée [sur les principes et valeurs] en vue de bien agir » (CEST 2017). L'éthique se distingue aussi de la déontologie qui « renvoie aux obligations que des personnes sont tenues de respecter dans leur travail » (CEST 2017). La déontologie permet parfois de trancher en cas de dilemme éthique (c'est-àdire de « conflits de valeurs ») en situation professionnelle.

L'éthique peut être abordée (1) d'un point de vue philosophique pour s'intéresser aux « fondements de la morale » ou (2) d'un point de vue pratique - on parle d'éthique appliquée. Celle-ci accompagne la prise de décision dans des situations concrètes qui présentent des enjeux particuliers autant en termes de processus que de la substance même des choix auxquels conduit la décision. La question éthique concerne à la fois l’individu et le groupe/la société (cf . Lafaye 2006).

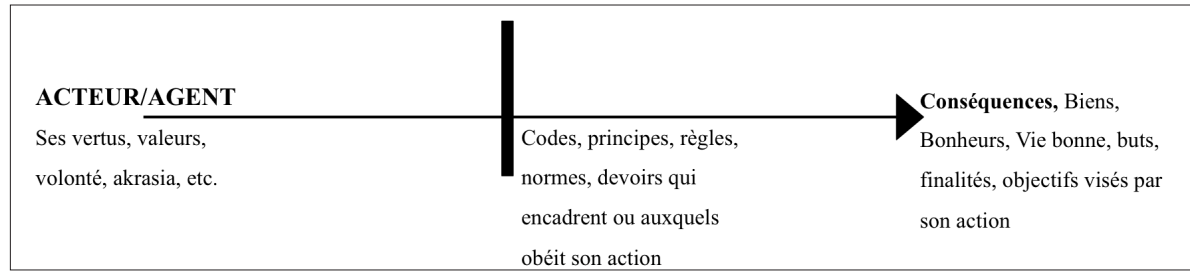

Figure 1. Logique de l'action éthique, d'après le schéma méthodologique global de l'action éthique (Bousbaci 2016).

$\mathrm{Au}$ Québec en particulier, « l'éthique appliquée est en effervescence » (CEST 2017). Dans le champ de l'aménagement, le milieu des praticiens a vu émerger depuis plusieurs décennies des paradigmes tels que le développement durable ou la participation citoyenne qui envisagent de poser les bonnes actions contribuant à la transformation de l'espace physique. Mais la référence à la notion d'éthique telle quelle semble récente.

1 Exemple la « morale chrétienne » 
M. Djemel, M. Ye, R. Bousbaci, G. Lizarralde I La temporalité du projet d'aménagement I DOI: 10.12977/ocula2019-4

L'enjeu de s'intéresser à l'éthique appliquée est notamment de comprendre comment les «dilemmes éthiques » peuvent être traités dans une situation pratique de prise de décision. Ces derniers interviennent lorsqu'il y a « conflits de valeurs », c'est-à-dire une "situation où les valeurs et les principes [auxquels un individu ou un groupe accorde de l'importance] entrent en opposition et rendent la décision difficile » (CEST 2017).

Pour résoudre un dilemme éthique, trois cas de figure se présentent (CEST 2016) : (1) hiérarchiser les principes et valeurs en contradiction puis (a) agir d'abord selon ceux qui semblent " primordiaux », tout en essayant de faire valoir les autres ou, (b) lorsque les principes et valeurs secondaires ne sont pas conciliables, trancher uniquement pour le principe primordial ou, (2) chercher un « compromis » entre les principes et valeurs lorsqu'il est impossible de les hiérarchiser. Si à l'échelle individuelle la résolution d'un dilemme éthique paraît simple, elle se complexifie quand plusieurs personnes interviennent dans les choix à effectuer en cas de conflit de valeurs. A ce titre, la (CEST 2017 ) affirme que : "Chacun a sa propre opinion sur les valeurs qui devraient guider les individus et la collectivité et chacun est libre de défendre celles auxquelles il tient. Différentes conceptions du bien commun, donc de la morale se côtoient. En contexte de pluralisme de valeurs, il n'est pas toujours facile de prendre des décisions collectives qui soient acceptables à tout un chacun. Dans une société confrontée à des enjeux de plus en plus complexes, l'éthique n'est pas une garantie qu'un consensus social émergera. Il s'agit plutôt d'une démarche pour prendre une décision éclairée en évaluant les arguments opposés. Cela s'incarne généralement dans des pratiques de délibération éthique. La principale difficulté reste souvent à déterminer, entre plusieurs valeurs importantes, laquelle ou lesquelles reflètent le mieux la société dans laquelle on veut vivre. L'éthique ne propose pas des solutions toutes faites à ce problème. Pour parvenir à une décision, la démarche éthique s'alimente d'arguments philosophiques, de consultations publiques, d'études scientifiques, d'avis d'experts, d'écrits intellectuels, etc. ».

Les conflits de valeurs sont assez courants dans les projets d'aménagement, car ces derniers sont particulièrement caractérisés par une diversité de parties prenantes qui défendent des intérêts différents, voire discordants et conflictuels. En effet, chaque partie prenante cherche à affirmer ses besoins et faire valoir la vision qu'elle a du projet. Certaines d'entre elles agissent dans le but de satisfaire les intérêts de l'organisation à laquelle elles appartiennent. Cette situation est encore plus marquante lorsque les points de vue exprimés dépassent le cadre des acteurs directement impliqués dans la démarche de projet pour inclure les habitants et les citoyens concernés de près ou de loin par ce dernier. Le comportement éthique des individus et groupes sociaux est souvent critiquable, notamment lorsque l'intérêt individuel (particulier) entre en contradiction avec l'intérêt commun. Le dilemme éthique survient aussi lorsque les parties prenantes ont une vision différente de ce qui fait intérêt commun. Il s'avère alors pertinent d'observer ces situations à la lumière des théories éthiques.

Notre approche consiste à analyser la confrontation entre les intérêts des parties prenantes dans le cas d'un projet de construction d'une école primaire. Nous mettons particulièrement en lumière les tensions qui surviennent entre les intérêts individuels défendus par des citoyens/habitants et ce qui repré- 
M. Djemel, M. Ye, R. Bousbaci, G. Lizarralde I La temporalité du projet d'aménagement I DOI: 10.12977/ocula2019-4

sente, a priori, un intérêt important pour la communauté : le projet d'une nouvelle école. Dans de telles situations, les parties prenantes se trouvent dans l'obligation de concilier (ou de trancher entre) des principes et valeurs sociaux d'une part (mœurs, déontologie, droit, morale) et individuels d'autre part (raisonnement éthique personnel) susceptibles de se trouver en contradiction.

L'exploration de ce sujet s'opère selon le schéma méthodologique global de l'action éthique (Fig.1). À travers ce modèle, nous voulons explorer la nature des actions entreprises par chaque partie prenante dans le projet.

Nous faisons en particulier l'hypothèse que ces tensions peuvent avoir une influence sur le processus de projet et les temporalités de ce dernier.

\section{Les temporalités du projet d'aménagement et le processus de prise de décision}

Plusieurs auteurs ont souligné une évolution significative dans le schéma des temporalités du projet. Le schéma classique linéaire, qui « procède de l'amont vers l'aval par une succession de phases bien identifiées et autonomes » (Janvier 2001 : 137), est aujourd'hui sujet à une transformation importante vers « une interaction réciproque » entre les phases amont et aval du projet. Cette transformation des modalités d'actions permet d'anticiper, dès l'amont du processus, les enjeux de financement et les contraintes futures de mise en œuvre opérationnelle, d'usage, de gestion et d'exploitation (d'après Bonetti 1998). Ce «bouleversement des rapports amont/aval » implique le « renouvellement des pratiques qui se cristallisent dans les phases amont » et qu'il convient d'analyser de plus près (Arab 2004 : 57). Entre autres renouvellements des pratiques, figure justement la participation citoyenne, qui a pour objectif d'inclure les citoyens dans le processus de prise de décision ; cette démarche pouvant amener à requestionner les décisions prises par les porteurs du projet et, de ce fait, prolonger le temps initialement prévu.

Or, la gestion du temps demeure un des enjeux de la conduite de projet, comme l'indique l'élaboration de nombreux outils de gestion du temps comme le diagramme de Gantt, ou le réseau PERT. Ces outils permettent notamment de préciser dès le départ une date de début et de fin des activités, l'enchainement de ces dernières, et d'anticiper les chemins critiques c'est-à-dire les activités qui ne doivent pas connaître de retard dans la démarche de projet.

Compte tenu de ce qui précède, on peut faire l'hypothèse que la démarche de participation citoyenne et son corollaire de controverses et de conflits de valeurs peuvent avoir une influence sur les temporalités du projet.

\section{Méthode : l'étude de cas d'une école primaire à l'île des sours à Montréal (Canada)}

\subsection{Contexte urbain et géographique, objectifs initiaux}

Le terrain proposé pour le projet d'école est situé à l'île des sœurs, dans un secteur résidentiel de l'arrondissement Verdun à Montréal. Ce dernier occupe une superficie générale de 9,83 km², et compte 66158 habitants en 2011 (Statistique Canada, recensement 2011). 


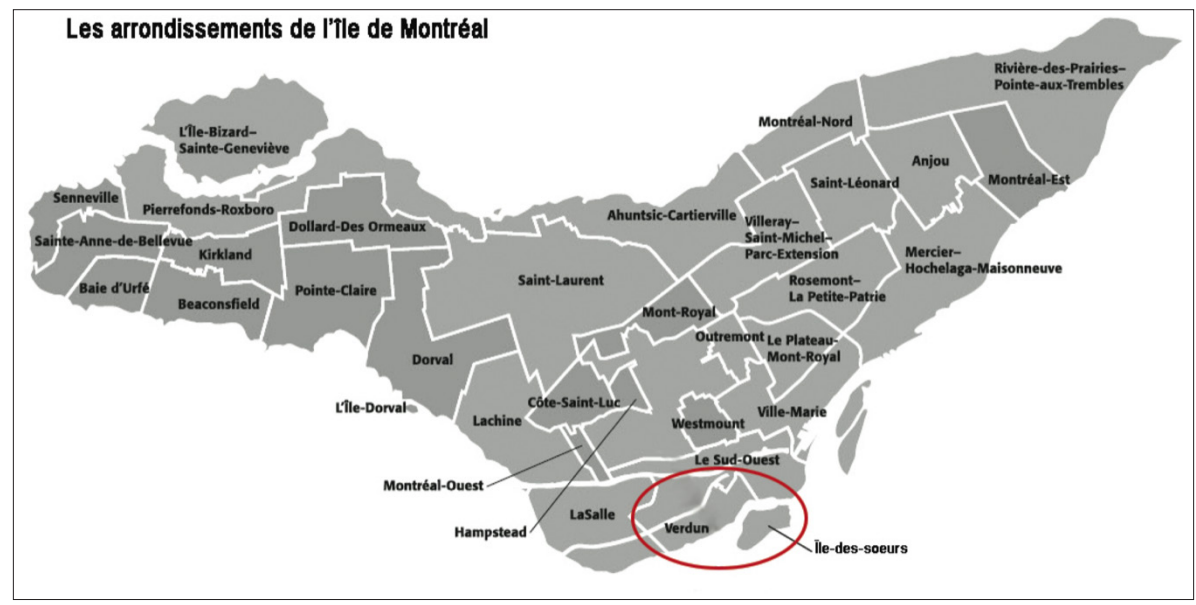

Figure 2. Plan de situation de l'arrondissement Verdun et de l'Île-des-Soeurs à Montréal. (Fond de carte <http://local.fiatlux.tk/montreal/image/arrondissements.jpg>, 2015)

Le projet consiste en la construction d'une école de 3 classes de maternelles et 18 classes primaires, avec un gymnase double vitré permettant de proposer aux résidents des services complémentaires aux terrains de tennis et à la piscine déjà existants (Commission scolaire Marguerite Bourgeois, 2014). L'arrondissement de Verdun a émis une série d'exigences ${ }^{2}$ pour l'autorisation du projet : (a) la construction sera sans ajout d'aires de stationnement et s'appuiera sur les principes de développement durable (b) le projet occupera une localisation sécuritaire favorisant les marcheurs (autobus pour les maternelles seulement), (c) le bâtiment aura occupation minimale au sol, (d) le projet favorisera le maintien des axes de circulation piétonnière en tout temps (e) des mesures de compensation seront prévues pour les arbres qui seraient abattus ou déplacés, (f) le projet favorisera le maintien de la vocation des équipements du parc, (g) le bâtiment aura une architecture de qualité en briques d'argile.

\subsection{Raison d'être et dates clés du projet}

En août 2011, l'arrondissement dépose le rapport du démographe, Norbert Robitaille, sur les prévisions de la population scolaire primaire à L'île-desSœurs. Le rapport confirme que les développements immobiliers de 2006 à 2011 ne semblent pas expliquer l'accroissement substantiel de la population scolaire qu'a connu l'école primaire de L'île-des-Sœurs (Ville de Montréal 2015). L'école primaire Elgar de l'île des sœurs, construite en 1985 (CSMB 2013), était considérée à la fin de l'année 2010 comme la plus grande école primaire au Québec, vu le nombre important d'élèves. En effet, elle a fait l'objet de trois agrandissements en 1993, 2002 et 2008. En 2011, l'école comptait près de 1 ooo élèves alors que la capacité maximale prévue par la commission scolaire est de 900 élèves (CSMB 2013).

2 Suite à une séance d’information tenue en juin 2013. 
M. Djemel, M. Ye, R. Bousbaci, G. Lizarralde I La temporalité du projet d'aménagement I DOI: 10.12977/ocula2019-4

Suite à cette situation alarmante, les parents étaient informés que certains élèves seraient transférés dans une école primaire de Verdun " terre-ferme », information qui a soulevé des contestations et des plaintes. L'école déclenchait alors des mesures de contingence pour que les élèves puissent rester sur place. Une demande avait déjà été présentée en 2010 par un groupe de parents d'élèves au MEL (Ministère de l'Éducation, des Loisirs et du Sport, aujourd'hui Ministère de l'Éducation et de l'Enseignement Supérieur) pour qu'une nouvelle école soit construite sur l'île. Finalement, c'est en 2011 que le Ministère de l'Éducation accorde l'autorisation de construire une nouvelle école.

\subsection{Cartographie des principales parties prenantes}

L'analyse de la structure organisationnelle du projet (à partir de documents) révèle trois groupes de parties prenantes tels qu'identifiés dans le tableau 1 : les parties prenantes politiques, professionnels de l'aménagement et les habitants et citoyens. Un quatrième groupe émerge, à savoir les médias. Cela est schématisé dans la figure 4 ci-après, i) les parties prenantes politiques à savoir le commanditaire, l'agent de contrôle, le donneur de l'ouvrage, l'arrondissement de Verdun, L'OCPM, le député de Verdun ii) les professionnels de l'aménagement à savoir l'industrie de la construction et les services d'aménagements et enfin iii) les habitants et citoyens regroupés dans des comités et groupes de pressions.

Le projet était soutenu par les parents et les élus qui y trouvaient un intérêt certain pour les enfants. Un groupe de riverains du parc s'opposait au projet sous prétexte de protéger le parc. Les médias ont influencé l'opinion publique vis-à-vis du projet en s'assurant de transmettre l'information objectivement (cf. Djemel, El-Bache, Marin-Valencia, \& Rivera 2015).

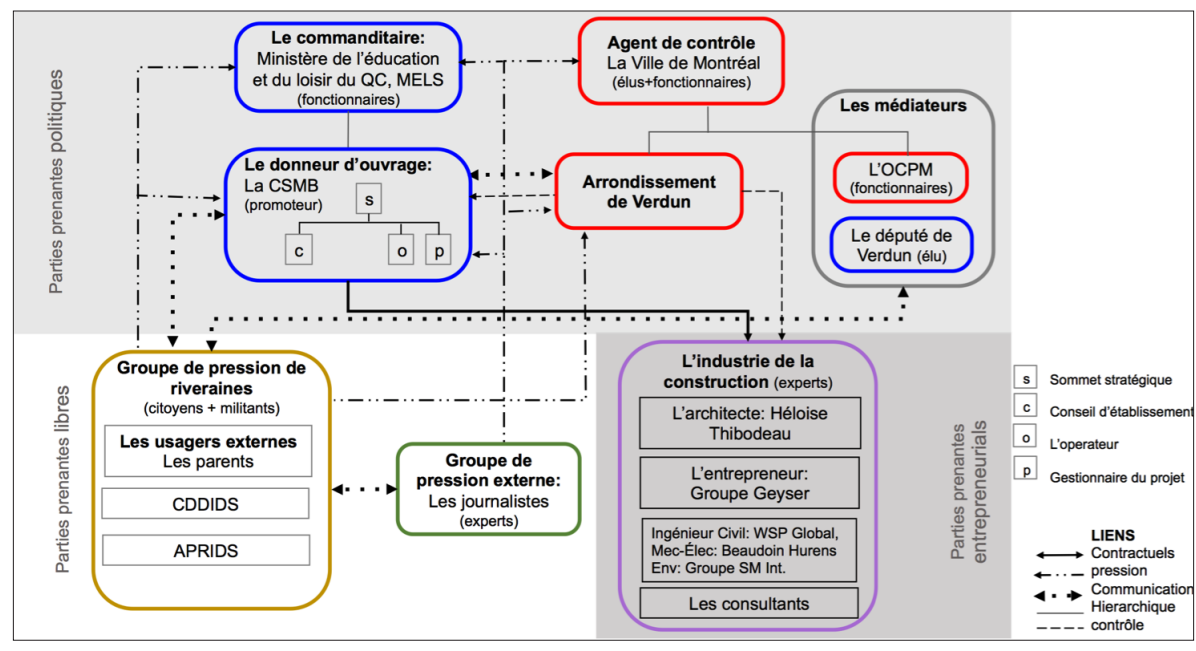

Figure 3. Diagramme organisationnel des parties prenantes du projet.

L'analyse documentaire montre que ces quatre groupes d'acteurs ont été influents dans la prise de décision, en soulevant notamment des tensions et 
M. Djemel, M. Ye, R. Bousbaci, G. Lizarralde I La temporalité du projet d'aménagement I DOI: 10.12977/ocula2019-4

des controverses lors de l'élaboration du projet de l'école. Nous focaliserons notre analyse en particulier sur quatre parties prenantes qui ont marqué les débats autour du choix du site du projet, principal sujet de controverse ; il s'agit de : i) la Commission scolaire Marguerite Bourgeois (CSMB), ii) l'arrondissement Verdun, iii) le comité parents et iv) Les groupes des riverains et le Comité pour le développement durable à L'île-des-Sœurs (CDDIDS).

\section{Résultats}

5.1. Lecture de la position des différentes catégories de parties prenantes à la lumière des courants de l'éthique

L'analyse de la position des parties prenantes clés dans le projet à la lumière des théories éthique identifiées au préalable nous aide à saisir les approches et positionnements éthiques de chacune d'elles afin de comprendre les tensions et controverses suscitées autour du projet.

L'exploration de ce sujet se fait selon le schéma méthodologique global de l'action éthique (fig.1). En appliquant ce modèle, nous cherchons à comprendre (a) la finalité ou la conséquence de l'action de chaque partie prenante ; (b) les devoirs et la déontologie qui guident l'action.

\subsection{La Commission scolaire Marguerite Bourgeois (CSMB)}

D'après la loi sur l'instruction publique du gouvernement du Québec (2019) les commissions scolaires sont des institutions présentes dans toutes les provinces du Canada. D'après le site officiel de la commission de Laval " ce sont de véritables institutions politiques locales possédant les attributs d'un gouvernement décentralisé doté de : i) une vie politique, ii) un pouvoir de taxation par l'impôt foncier, iii) une juridiction sur un territoire propre, iv) d'une responsabilité devant la population locale par l'élection des commissaires au suffrage universel. La responsabilité première de la commission scolaire consiste à s'assurer que les personnes relevant de sa compétence reçoivent les services éducatifs auxquels elles ont droit.

La deuxième responsabilité importante consiste à répartir équitablement les ressources entre ses établissements, en tenant compte des besoins exprimés et des inégalités sociales et économiques. La troisième responsabilité consiste à organiser et à offrir des services à la communauté. Ces services peuvent être de nature culturelle, sociale, sportive, scientifique ou communautaire, comme les services de garde, les activités de formation de la main-d'oeuvre, les services aux entreprises et les activités de coopération avec d'autres organismes du milieu. À ces différentes activités s'ajoutent d'autres champs d'action qui contribuent activement au développement local et régional : la participation à différentes instances de développement local et régional ; la mise en place d'infrastructures (fibre optique, gymnase, piscines, terrains de jeux, etc.) » (CSLAVAL 2016). 
M. Djemel, M. Ye, R. Bousbaci, G. Lizarralde I La temporalité du projet d'aménagement I DOI: 10.12977/ocula2019-4

Tableau 2. Analyse de l'action éthique de la CSMB.

\begin{tabular}{ll}
\hline Variables & Analyse de l'action \\
& $\begin{array}{l}\text { Permettre aux résidents de l'Île-des-sœurs d'avoir accès à une école } \\
\text { primaire à proximité. }\end{array}$ \\
Offrir un cadre propice pour le développement et l'éducation des \\
enfants. \\
La commission scolaire a procédé en concordance avec les normes \\
et les valeurs juridiques stipulées par les codes et les textes de lois \\
régissant ce type de projet.
\end{tabular}

\subsection{L'arrondissement de Verdun}

Tel qu'apparaît sur la figure 3, Verdun est un arrondissement de la ville de Montréal, situé au sud-ouest de l'île entre la rive du fleuve Saint-Laurent en aval des rapides de Lachine et le Canal de l'Aqueduc. Délimité à l'Est par le Pont Champlain et l'Autoroute 15 et à l'Ouest par l'arrondissement de LaSalle, il inclut également l'île-des-Soeurs.

Tableau 3. Analyse de l'action éthique de l'arrondissement de Verdun.

\begin{tabular}{ll}
\hline Variables & Analyse de l'action \\
& Se trouvant dans une position délicate, subissant la pression de la \\
commission scolaire et du comité parent l'objectif ultime était de \\
trouver un terrain convenable et adéquat au projet. \\
Étant une institution publique le rôle de l'arrondissement de Verdun \\
est d'assurer le bien-être et le bon fonctionnement de la vie dans \\
l'arrondissement. \\
L'objectif de l'arrondissement est donc de trouver le consensus des \\
citoyens dans les décisions les concernant et de leur offrir un cadre de \\
vie adéquat aux citoyens afin qu'ils puissent jouir et sépanouir, être \\
engagé politiquement et socialement dans l'arrondissement. \\
Dans le cadre de ce projet la conception du 'bien-agir' de l'arrondisse- \\
ment de Verdun est de trouver un compromis face au conflit généré \\
par le choix du site de la nouvelle l'école, à travers les consultations, \\
les révisions et les comités détudes offrir un cadre de connaissance et \\
remise en question. \\
Une consultation publique menée par l'oCPM a été réalisée, des \\
séances d'information, des comités formés par l'arrondissement pour \\
favoriser une meilleure informations sur le projet.
\end{tabular}

\subsection{Le comité des parents : Dessine-moi une école}

C'est un regroupement de parents qui a initié le projet en 2010 en déposant une demande à la CSMB pour une nouvelle école primaire sur l'île des Soeurs. Au début, ils s'organisaient dans le comité dessine moi une école, qui s'est transformé en l'Association des Familles de l'île des Sours (AFIDS) (cf. Zerda 2015). La relève dans la négociation avec la CSMB est prise pour un deuxième groupe de parents, le Collectif Besoin-école qui à la fin réussirait à obtenir le terrain nommé 'le triangle" pour la construction (cf. Zerda 2015).

Dans son rapport de consultation publique, l'Office de consultation publique de Montréal affirme que l'association des familles de l'île des Soeurs (AFIDS), 
M. Djemel, M. Ye, R. Bousbaci, G. Lizarralde I La temporalité du projet d'aménagement I DOI: 10.12977/ocula2019-4

fondée en avril 2012 « dans la foulée du débat entourant le projet de construction d'une seconde école primaire, a fait valoir son point de vue. L'association, qui compte plus de 500 membres, est impliquée dans les projets susceptibles d'avoir un impact sur la qualité de vie des familles de l'île. Le comité des parents Dessine-moi une école, qui milite depuis 2011 pour la construction de l'école, a également donné son opinion sur le projet » (OCPM 2013 : 11). Nombreuses actions ont été entreprises par les parents et les meneurs de ce mouvement afin de faire valoir l'urgence et le besoin du projet. Leurs activités consistaient à l'organisation d'événements de socialisation dans le voisinage en invitant journalistes et activistes, des groupes de pressions dans les réseaux sociaux, des discours dans les radios et les journaux locaux, etc.

Tableau 4. Analyse de l'action éthique du comité dessine-moi une école.

\begin{tabular}{ll}
\hline Variables & Analyse de l'action \\
& La formation de ce comité émane du besoin pressant de trouver \\
une solution urgente, afin d'éviter de déplacer les élèves de lécole \\
primaire à l'extérieur de l'île. Motivé par un financement accordé par \\
le mouvementent ce comité a exercé une pression énorme sur la com- \\
mission scolaire, l'arrondissement ainsi que les résidents de l'île afin \\
d'accélérer le processus de prise de décision. La conséquence de ce \\
mouvement est certes moralement bonne, vu qu'elle vise l'édification \\
d'une institution éducative. L'enjeu non reconnu par ce comité étant \\
celui de sacrifier un espace vert pour l'accomplissement du projet. \\
Le comité des parents est un ensemble d'associations et d'activistes de \\
la société civile qui agissent en faveur de la réalisation de l'école. Afin \\
d'être reconnus, ils sont représentés dans des associations et des re- \\
groupements reconnus et enregistrés. Ils opèrent d'une manière légale \\
et conforme aux lois qui encadrent leurs actions dans ce projet.
\end{tabular}

\subsection{Les groupes des riverains ou le Comité pour le développement durable à L'île-des-Sœurs (CDDIDS)}

Dans leur mémoire présentée à l'OCPM, le Comité pour le développement durable de L'Île des Soeurs (CDDIDS), était « fondé en mai 2011 suite à l'annonce du projet d'école sur le terrain de stationnement du parc de la Fontaine, le CDDIDS représente les 747 signataires du registre pour la tenue d'un référendum en 201 ». (CDDIDS $2013: 12$ ). Ce comité qui compte près de 800 adhérents (CDDIDS 2013), est une entité qui est « à l'origine de la campagne de financement Adoptez un arbre menée en 2012 afin de recueillir les fonds nécessaires pour intenter un éventuel recours judiciaire. Le comité se consacre à la protection de l'environnement et du milieu de vie des résidents, et veille à l'application des principes de développement durable dans les développements de l'île. » (OCPM $2013: 12)$.

À travers le mémoire présenté à l'OCPM, ils voulaient insister sur le fait que « le CDDIDS souhaite la construction de cette deuxième école primaire publique dans les meilleurs délais possible, mais s'oppose au choix du site proposé. On a prétendu et certaines personnes continuent à prétendre ou à croire qu'il n'y a pas d'autres endroits pour construire cette école alors qu'il existe au 
M. Djemel, M. Ye, R. Bousbaci, G. Lizarralde I La temporalité du projet d'aménagement I DOI: 10.12977/ocula2019-4

moins trois sites alternatifs à L'île-des-Soeurs. » (CDDIDS 2013 : : 18). Le discours du CDDIDS soulève « une lutte pour la préservation de tout parc à L'Îledes-Soeurs et continue à mobiliser toutes les forces vives soucieuses du développement durable contre tout empiètement des espaces verts et du milieu de vie des résidents et résidentes de L'île-des-Soeurs. » (CDDIDS $2013: 19$ ).

Tableau 5. Analyse de l'action éthique des groupes riverains.

\section{Variables}

Conséquences

Devoirs

\section{Analyse de l'action}

Lobjectif ultime de ce groupe était de bloquer la réalisation du projet sur le terrain spécifié par la ville, en loccurrence le parc Lafontaine. Dans leurs discours ils affirment qu'ils ne sont pas contre l'idée du projet décole, mais contre son emplacement géographique et défendent aussi leur perception du bien agir. Leur argument est en particulier de préserver la quiétude des riverains du parc contre déventuels bruits que provoqueraient les élèves dans le cours de lécole, et de maintenir le rôle environnemental que jour le parc sur l'île.

Limplication de ce groupe d’acteur fait partie de leur droit de participation et de paroles. Alors, afin doptimiser leurs actions ils se sont organisés en associations civiles, qui agissait conformément à leurs normes et valeurs déontologiques.

\section{L'influence du rôle des parties prenantes sur la temporalité du projet}

Par analogie aux projets scientifiques et de laboratoires, la temporalité des projets pourrait être définie telle qu'identifiée dans les recherches de Brunet et Dubois qui en distinguent différents types « entendus comme l'inscription dans le temps de différentes séquences d'action - pour rendre compte de l'évolution des collectifs de recherche » (Brunet et Dubois 2012 : 2).

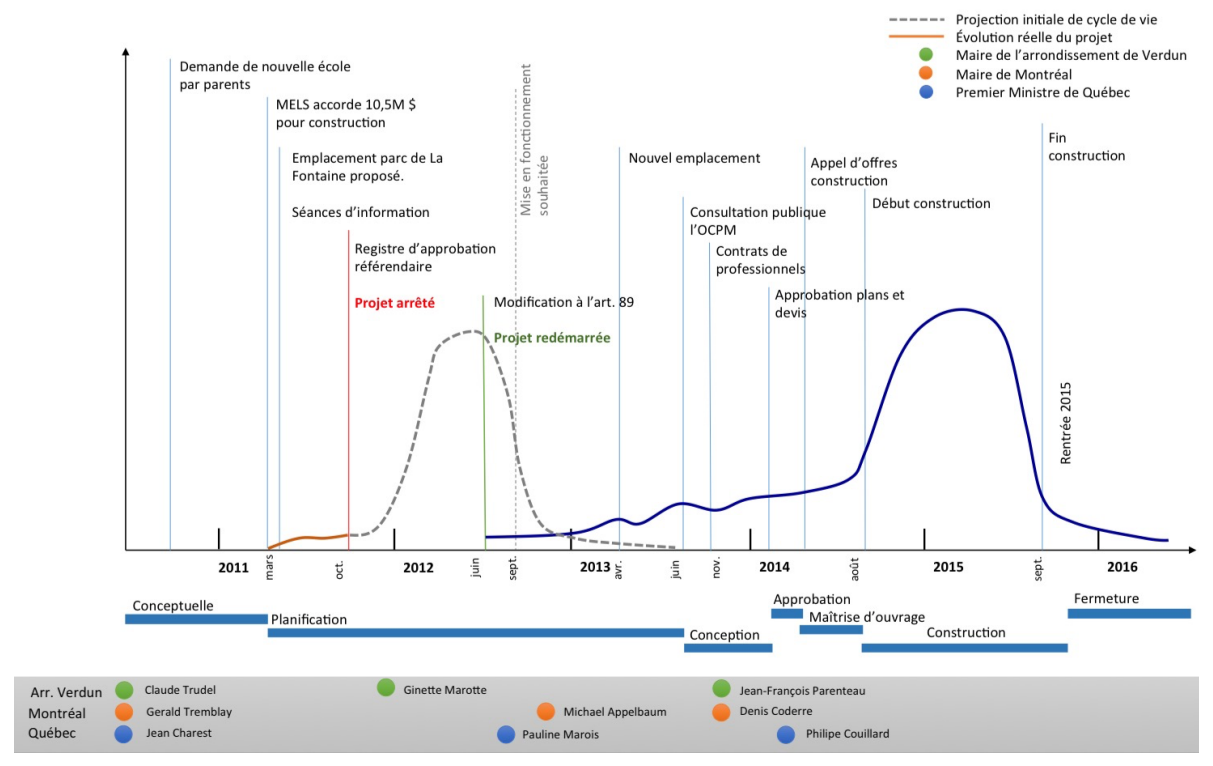

Figure 4. Diagramme temporel indiquant les étapes charnières du projet de construction d'une nouvelle école sur l'île-des-Soeurs (Montréal, Canada). 
M. Djemel, M. Ye, R. Bousbaci, G. Lizarralde I La temporalité du projet d'aménagement I DOI: 10.12977/ocula2019-4

Dans ce travail sur l'émergence d'un institut de recherche biomédicale, Brunet et Dubois identifient trois types de temporalités (cf. Schultz 2013) : une temporalité professionnelle caractérisée par « des pas de temps longs et surtout peu prévisibles » qui illustre la période de conceptualisation et de planification dans les projets d'aménagement ; une temporalité normative ou sociétale, telle que les délais des législations ; et enfin une temporalité gestionnaire courte qui « formalise le déroulement temporel du projet » (Brunet $2012: 2$ ).

La figure 6 montre clairement l'impact de la participation citoyenne sur le processus du projet. L'arrêt du projet était entre autre du au désaccord entre les parties prenentes et l'opposition manifestée par un groupe signficatif de riverains qui refusaient l'implantation du projet dans leur voisinage. Cela nous rappelle la notion not in my backyard, ou pas dans ma cour qui s'exprime par " l'opposition de la population locale à l'encontre de l'implantation d'équipement ou de groupes d'individus considérés comme nuisibles » (Marchetti 2005:5).

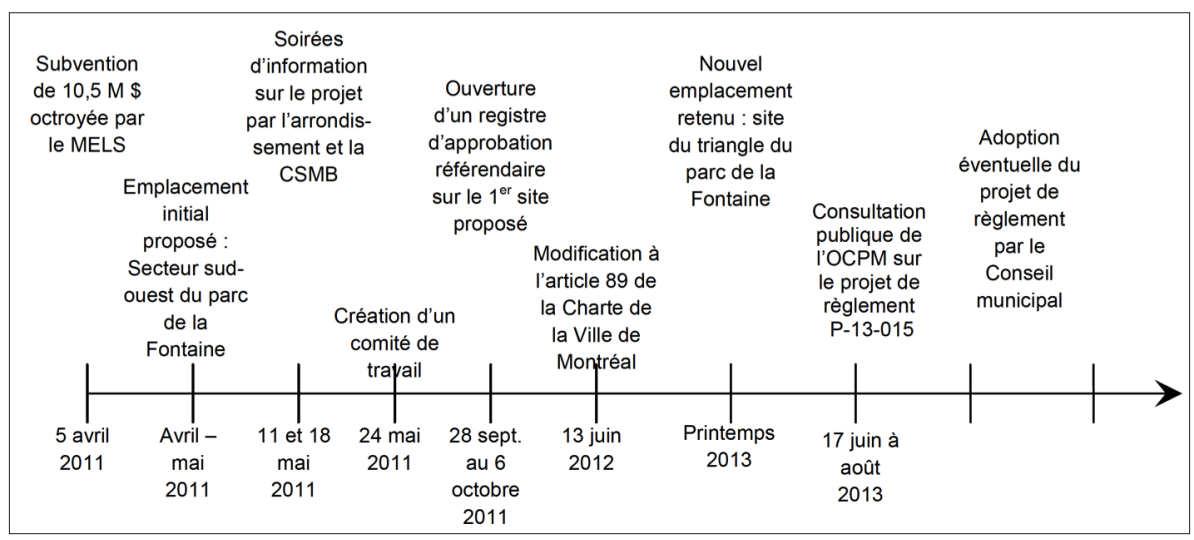

Figure 5. Cheminement des différentes étapes du projet (OCPM 2013)

Entamé en mars 2011 avec l'approbation du MELS (Ministère de l'éducation, des loisirs et des sports) d'accorder un budget à la construction de la nouvelle école sur l'île-des-sœurs, le projet a connu un arrêt dès octobre 2011, dû à l'opposition d'un groupe de riverains et du comité pour le développement durable de l'île-des-sœurs. Ces deux parties prenantes ont en particulier bloqué (au cours de la séance d'information et de consultation ) l'approbation au changement de zonage du parc La Fontaine en vue d'y construire l'école. Ce blocage a amené l'arrondissement Verdun à mettre en place un comité de travail pour bonifier le projet, à défaut de proposer à la commission scolaire d'autres alternatives de terrains à construire.

Finalement, les travaux du comité ont permis de proposer, en avril 2013, un nouvel emplacement pour la construction de l'école ; mais il ne s'agit pas de réelles alternatives puisque la solution reste d'occuper une partie de parc : plutôt que de construire sur le stationnement, l'école sera édifiée sur le triangle. Malgré l'opposition de riverains et du CDDIDS, l'arrondissement a refusé de proposer d'autres terrains, indiquant qu'elle n'en disposait pas. 
M. Djemel, M. Ye, R. Bousbaci, G. Lizarralde I La temporalité du projet d'aménagement I DOI: 10.12977/ocula2019-4

Les acteurs de ce groupe défendaient avec ferveur le maintien du parc Lafontaine et la non-édification de l'école sur le site proposé par l'arrondissement. Toutefois, nombreux facteurs et considérations tant politiques que sociales ont contribué à réaliser le projet et ainsi ne pas répondre de ce groupe.

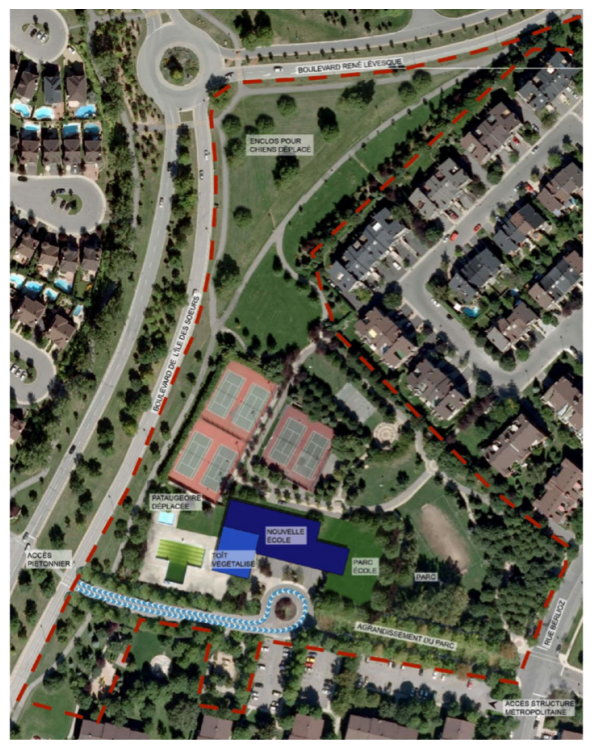

Figure 6. 1ère proposition de la nouvelle école.

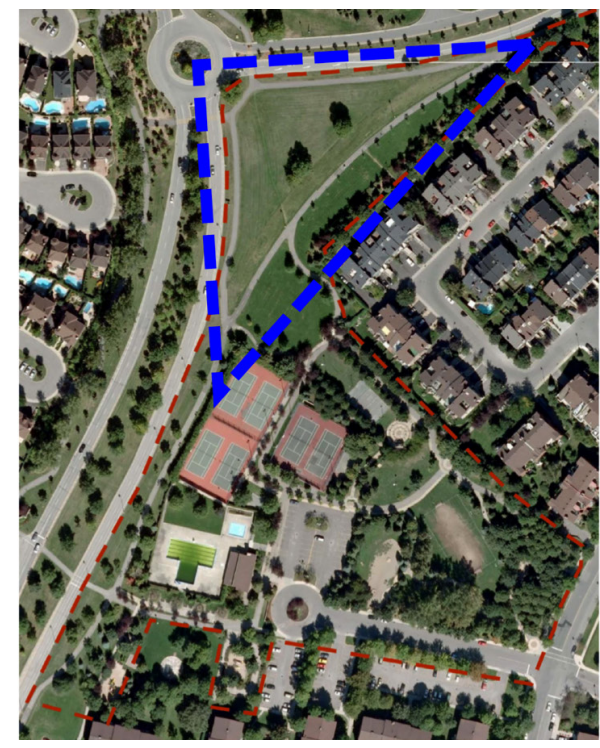

Figure 7. Le site retenu.

\section{Discussion}

\subsection{La participation publique, révélatrice de tensions entre les différentes parties prenantes}

Le projet d'école est initié par la commission scolaire et soutenu par l'arrondissement Verdun. L’intérêt commun parait alors une évidence pour ces acteurs : accompagner l'éducation des enfants de l'île et leur éviter de longs trajets pour aller étudier sur la terre ferme, à savoir un voyage de près d'une heure en autobus scolaire. Mais la participation citoyenne révèle qu'il existe deux perceptions différentes de l'intérêt commun porté par deux groupes de citoyens : l'idée de construire une école semble unanime chez les habitants de l'île, mais elle n'occupe pas la même place pour tous dans la hiérarchie des valeurs de ce qui relève de l'intérêt commun. Les parents d'élève estiment en effet que l'école est la valeur primordiale. On peut sacrifier une partie des terrains du parc urbain pour la construire sur ce dernier. A contrario pour les riverains de ce parc, ce dernier constitue une valeur à maintenir dans le quartier. Il l'est d'autant plus qu'il contribue à maintenir le calme dans le voisinage alors que la présence d'une école est source d'achalandage de voitures et de bruit d'élèves dans la cour de récréation.

Cette situation est clairement un dilemme éthique où plusieurs principes et valeurs de l'intérêt commun entrent en conflit ; elle interroge également la manière avec laquelle cela peut être résolu. 
M. Djemel, M. Ye, R. Bousbaci, G. Lizarralde I La temporalité du projet d'aménagement I DOI: 10.12977/ocula2019-4

7.2. De la participation citoyenne « excessive » vers une construction collective de valeurs partagées : concilier les arguments divergents et favoriser des apprentissages réciproques

Comme développé dans le cadre conceptuel, le dilemme éthique peut être résolu de trois manières : (1) hiérarchiser les valeurs et opter pour la première dans la prise de décision, tout en conciliant cette dernière avec les autres valeurs considérées comme non primordiales dans la hiérarchie ; (2) considérer uniquement la valeur primordiale lorsque cette dernière est inconciliable avec les autres ; (3) chercher un compromis entre toutes les valeurs lorsqu'il est impossible de les hiérarchiser.

Dans le cas à l'étude, la question est de savoir s'il est réellement possible de hiérarchiser les valeurs et qui est en mesure de le faire. Construire une école primaire semble un projet d'intérêt commun, mais conserver le parc pour des questions environnementales en général et pour le bien être des personnes retraitées vivant à proximité peut bien s'inscrire dans une logique du bien commun. Mais comme le souligne la commission québécoise de l'éthique en science et technologie, "l'éthique n'est pas une garantie qu'un consensus social émergera. Il s'agit plutôt d'une démarche pour prendre une décision éclairée en évaluant les arguments opposés ». Cette considération nous semble particulièrement importante à prendre en considération dans le processus de projet pour éviter les ralentissements ou les blocages dus à des controverses et des conflits de valeurs. Adopter un schéma linéaire de temporalité de projet, où les activités et les interventions des acteurs sont successives, c'est prendre le risque de faire remettre en question à un moment du processus les décisions qui ont été prises au préalable, même si ces dernières peuvent se justifier. La participation peut, dans ce contexte, être un frein à la temporalité du projet et à sa mise à œuvre et être considérée comme excessive si elle intervient tardivement ${ }^{3}$ dans le processus du projet.

On peut ainsi avoir un autre angle d'analyse des conséquences de la participation citoyenne sur le processus : plutôt que de la pointer du doigt (comme étant excessive), on peut interroger (1) la place qu'elle a dans la démarche de projet et (2) sa portée dans la planification/conception réelle. Autrement dit, est-ce que les habitants/citoyens sont simplement informés des choix déjà opérés sans avoir réellement de marge de manœuvre ? Ou sont-ils considérés comme des acteurs à part entière de la démarche de projet qui (a) sont impliqués à chacune des étapes du projet, y compris les plus en amont et qui (2) peuvent avoir une voix dans les choix à opérer. Nous pensons que la participation peut être effective et paraitre moins abusive si elle est pensée dans le cadre de « l'agir communicationnel » tel que conceptualisée par Habermas. Cela consiste à « construire une relation entre sujets qui repose sur un langage partagé » (Paquot 2008 : xiii). La notion de langage partagé nous semble être la clé. Ce langage partagé concerne à la fois (a) les règles et procédures de

3 Dans le cas de figure, les citoyens sont consultés après avoir choisi le terrain alors qu'ils auraient pu être impliqués dès l'idée de construire une école afin de dialoguer sur les opportunités qui se présentent. 
M. Djemel, M. Ye, R. Bousbaci, G. Lizarralde I La temporalité du projet d'aménagement I DOI: 10.12977/ocula2019-4

la démarche de projet ; qui précise les (i) modalités d'implication des différents acteurs (y compris les habitants/citoyens) et (ii) la façon dont les choix d'aménagement se feront. Dans le champ de l'aménagement urbain, certains chercheurs ont conceptualisé cette démarche de l'agir communicationnel en " programmation (urbaine ou architecturale) inclusive et participative » (Zetlaoui-Léger, 2010, 2013). Cette démarche consiste à co-élaborer le projet à ses différentes étapes, en commençant par un diagnostic partagé de la situation actuelle qui recueille les points de vue et optimise le choix du site et trouver des compromis acceptables lorsqu'il n'y a pas de consensus.

Cette démarche est particulièrement le lieu d'apprentissages réciproques entre les acteurs du projet qui échangent sur les éléments à développer. Ainsi, les porteurs du projet apprennent des citoyens leurs besoins, notamment ceux qui n’avaient pas été anticipés ; les citoyens non organisés apprennent des porteurs du projet les contraintes pour lesquels les marges de manœuvre sont faibles et proposent des alternatives acceptables pour eux.

\section{Conclusion}

La participation citoyenne est un paradigme de prise de décision qui vise la démocratie directe et la justice sociale. Mais elle peut donner l'impression d'être abusive lorsqu'elle conduit à des blocages dus à l'expression de divergences, voire de conflits entre plusieurs principes et valeurs de ce qui fait l'intérêt de la collectivité. L'approche de ces situations sous l'angle de l'éthique permet notamment de mettre en lumière les ambitions qui animent les différentes parties prenantes pour ensuite envisager la manière dont ces tensions peuvent être atténuées. Elle fournit des pistes de résolutions de conflits, mais interroge les conditions de négociations : quels sont les pré-requis pour favoriser des compromis acceptables?

\section{Bibliographie}

Arab, Nadia

2004 L'activité de projet dans l'aménagement urbain : processus d'élaboration et modes de pilotage Les cas de la ligne $B$ du tramway strasbourgeois et d'Odysseum à Montpellier, École des Ponts ParisTech.

Aristote

Éthique à Nicomaque, trad. Jean Tricot, Paris : Vrin, 1983, trad. Première édition 1856

André Thibault, Marie Lequin et Mireille Tremblay

2000 Cadre de référence de la participation publique (démocratique, utile et crédible), Québec, Conseil de la santé et du bien-être.

Bousbaci, Rabah

2016 Cours Théories du projet AME6817, Faculté de l'aménagement, université de Montréal, Hiver2016. 
M. Djemel, M. Ye, R. Bousbaci, G. Lizarralde I La temporalité du projet d'aménagement I DOI: 10.12977/ocula2019-4

Brunet, Philippe ; Dubois, Michel

2012 «Cellules souches et technoscience : sociologie de l'émergence et de la régulation d'un domaine de recherche biomédicale en France », Revue française de sociologie, 53 (3), p. 391-428.

CEST : Commission de l'éthique, en science et en technologie, (site web gouvernemental officiel),: <http://www.ethique.gouv.qc.ca/fr/commission/ la-commission.html>. Consulté en 2017.

Cherns, Albert. B., \& Bryant, Donald. T.

1984 «Studying the client's role in construction management», Construction Management and Economics, 2(2), pp. 177-184. DOI : 10.1080/01446198400000016.

CSL : Commission scolaire de Laval (site web officiel) <https://www2.cslaval.qc.ca/spip.php?article249>. Consulté en mai 2017.

Côté, L., Fung, A., Fischer, F., Gauthier, M., Simard, L., Fourniau, J.-M., Venne, M.

2011 «La participation citoyenne », Télescope Revue d'analyse comparée en administration publique, Vol. 17 (n. 1). 229p.

Djemel, Manel; El-Bache, Dany; Marin-Valencia, Philippe; \& Rivera, Wendy; 2015 «Une nouvelle école à L'Île-des-Sœurs : de la négociation à la réalisation», AME 6916 : Gestion des parties prenantes, Faculté de l'aménagement, université de Montréal.

Freeman, R. Edward

1984 Strategic Management : A Stakeholder Approach, Boston, Pitman.

Gardesse, Camille

2011 «La concertation citoyenne dans le projet de réaménagement du quartier des Halles de Paris (2002-2010), Les formes de la démocratisation de l'action publique en urbanisme et ses obstacles», Thèse de doctorat en Urbanisme, Aménagement et Politiques urbaines de l'Université Paris Est Créteil, Lab'Urba, dir ${ }^{\circ}$ Zetlaoui-Leger J.

Green, S. D.

1996, A metaphorical analysis of client organizations and the briefing process. Construction Management and Economics, 14(2), 155-164. DOI : 10.1080/014461996373593

Hemmati, Minu

2002 «Multishtaholders processes of governance and sustainability: Beyoung Deadlbock and conflict», Londres : Earthscan. Hare, A.

Howard, Ebenzer

1965 «Garden cities of to-morrow», Cambridge, Mass : The M.I.T. Press.

Janvier, Yves

2001 «Un système de production en mutation », in Masboungi Ariella (dired..), Fabriquer la ville. Outils et méthodes : les aménageurs proposent. Paris, La Documentation française, pp. 137-147.

Lafaye, Caroline Gilbert

2006 Justice sociale et éthique individuelle, Collection : Inter-Sophia, 456p, ISBN : 2-7637-8365-1 
M. Djemel, M. Ye, R. Bousbaci, G. Lizarralde I La temporalité du projet d'aménagement I DOI: 10.12977/ocula2019-4

Lizzarlde, Gonzalo ; Djemel, Manel

2010 «La gouvernance des projets d'architecture : Une typologie de la multiorganisation temporaire», Les ateliers de l'éthique, 5(2), 76-87.

Marchetti, Nicolas

2005 «Les conflits de localisation : Le syndrome NIMBY», Rapport Bourgogne (CIRANO), 1, $32 \mathrm{p}$.

OCPM : Office de consultation publique de Montréal

2013 «Rapport de consultation publique : Projet de construction d'une école primaire à l'île des sœurs», Projet de règlement $P$. 13, 015, Montréal.

Pinson, Gilles

2009 Gouverner la ville par projet : urbanisme et gouvernance des villes européennes, Paris : Presses de Sciences Po.

PMI Book, (Project management institute book)

2013 A guide to the project management body of knowledge: (PMBOK $囚$ guide) (5th ed.). Newtown Square, Pa. : Project management institute.

Steve, Rowlinson; Peter, McDermott

1999 Procurement Systems: A Guide to Best Practice in Construction, Routledge 342 Pages, ISBN 9780419241003

Schultz, Émilien

2013 «Le temps d'un projet », Temporalités, <http://journals.openedition.org/ temporalites/2563> ; DOI : 10.400o/temporalites.2563. Consulté le 12 avril 2019 .

Viel, Laurent ; Lizarralde, Gonzalo ; Maherzi, Fella ; Thomas-Maret, Isabelle

2012 «L'influence des parties prenantes dans les grands projets urbains. Les cas du Quartier des spectacles de Montréal et de Lyon Confluence», Cybergeo : European Journal of Geography.

Zetlaoui-Léger, Jodelle.

2013 La concertation citoyenne dans les projets d'écoquartiers en France : évaluation constructive et mise en perspective européenne, Tome1, Lab’Urba, Université Paris Est Créteil-Val de Marne.

Zetlaoui-Léger, Jodelle

2010 Programmation architecturale et urbaine, L'abc de l'urbanisme. I.d.u.d. Paris, Université Paris Est Créteil. 